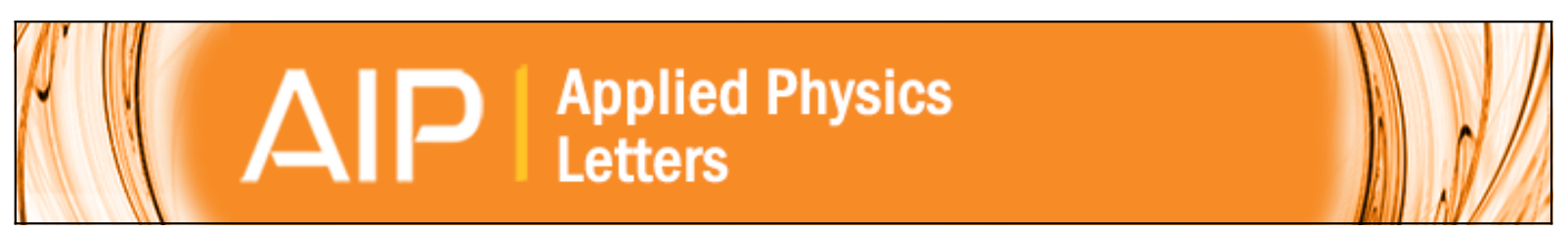

InP quantum dot lasers with temperature insensitive operating wavelength

S. Shutts, P. M. Smowton, and A. B. Krysa

Citation: Applied Physics Letters 103, 061106 (2013); doi: 10.1063/1.4817732

View online: http://dx.doi.org/10.1063/1.4817732

View Table of Contents: http://scitation.aip.org/content/aip/journal/apl/103/6?ver=pdfcov

Published by the AIP Publishing

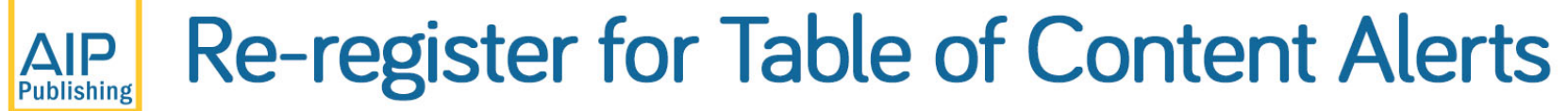

Create a profile.

Sign up today! 


\title{
InP quantum dot lasers with temperature insensitive operating wavelength
}

\author{
S. Shutts, ${ }^{1}$ P. M. Smowton, ${ }^{1}$ and A. B. Krysa ${ }^{2}$ \\ ${ }^{1}$ School of Physics and Astronomy, Cardiff University, Cardiff CF24 3AA, United Kingdom \\ ${ }^{2}$ EPSRC National Centre for III-V Technologies, Department of Electronic and Electrical Engineering, \\ University of Sheffield, Mappin Street, Sheffield S1 3JD, United Kingdom
}

(Received 16 May 2013; accepted 21 July 2013; published online 6 August 2013)

\begin{abstract}
We quantify the mechanisms that govern the lasing wavelength in edge-emitting InP/AlGaInP quantum dot (QD) lasers, by characterising the constituent factors controlling the temperature dependence of the gain peak wavelength. We show that a regime exists where the temperature coefficient of the bandgap can be compensated by the increasing wavelength-shift associated with state-filling in the QD ensemble, necessary to recover the gain peak magnitude. We demonstrate cleaved-facet edge-emitting lasers with a wavelength temperature dependence of $0.03 \mathrm{~nm} / \mathrm{K}$, similar to the temperature dependence of a Bragg stack fabricated in this material and approximately a sixth of the dependence of the bandgap. (C) 2013 AIP Publishing LLC. [http://dx.doi.org/10.1063/1.4817732]
\end{abstract}

The InP QD laser structures were grown by low pressure metal-organic vapour phase epitaxy (MOVPE) on n-GaAs (100) substrate oriented $10^{\circ}$ toward $\langle 111\rangle$. Samples were grown at $730^{\circ} \mathrm{C}$ using trimethyl precursors for the group III elements and arsine $\mathrm{AsH}_{3}$ and phosphine $\mathrm{PH}_{3}$ as precursors of the group V elements. Self-assembled dots were formed from the equivalent of 3 monolayers of InP material deposited on $\left(\mathrm{Al}_{0.3} \mathrm{Ga}_{0.7}\right)_{0.51} \mathrm{In}_{0.49} \mathrm{P}$ and covered with $8 \mathrm{~nm}$ $\mathrm{Ga}_{0.54} \mathrm{In}_{0.46} \mathrm{P}$ QWs. Using a composition of $\mathrm{Ga}_{\mathrm{x}} \mathrm{In}_{1-\mathrm{x}} \mathrm{P}$ that is tensile strained compared to the underlying substrate reduces the current density required to achieve lasing on the QD states as described in Ref. 1. Structures contained 5 layers of dots in wells (D-WELL), where each (D-WELL) layer was separated by $16 \mathrm{~nm}$ wide $\left(\mathrm{Al}_{0.3} \mathrm{Ga}_{0.7}\right)_{0.51} \mathrm{In}_{0.49} \mathrm{P}$ barrier layers. The rest of the waveguide structure is formed from $1000 \mathrm{~nm}$ wide $\mathrm{Al}_{0.51} \mathrm{In}_{0.49} \mathrm{P}$ cladding layers, doped with $\mathrm{Si}$ and $\mathrm{Zn}$ for $\mathrm{n}$ and $\mathrm{p}$-types, respectively.

In these QD structures, the inhomogeneous broadening and the separation between the ground state (GS) and excited state (ES) was determined from measured modal absorption spectrum. The GS-ES separation was found to be $50 \mathrm{meV}$, which is less than that found for InAs QDs emitting at $1.3 \mu \mathrm{m}$, where the separation is typically $60-80 \mathrm{meV}^{2}$ The inhomogeneous broadening (determined by fitting a Gaussian function to the GS absorption), has a value $(\sigma)$ of $24 \mathrm{meV}$ compared to, for example, a GS broadening of $30 \mathrm{meV}$ as previously determined for InAs QDs. ${ }^{4}$

Samples were fabricated into $50 \mu \mathrm{m}$ wide oxide-isolated stripe multi-section test structures and lasers. All devices have uncoated as-cleaved facets and were operated pulsed, with a pulse length of $1000 \mathrm{~ns}$ and a duty cycle of $0.1 \%$ to avoid self-heating. Measurements using the segmented contact method allow us to determine the net modal gain spectra and the optical loss spectra, the latter being the sum of the modal absorption and the internal optical mode loss, $\alpha_{\mathrm{i}}{ }^{3}$

Example optical loss and modal gain spectra that illustrate the mechanisms involved are plotted in Figure 1. The optical loss spectra, where we focus on the long wavelength region corresponding to transitions on the low energy edge of the dot distribution, are plotted as negative gain. As expected, the whole absorption edge red-shifts with increasing temperature from $240 \mathrm{~K}$ to $300 \mathrm{~K}$ and this reflects the shift in energy of the allowed dot states caused by the change in energy of the constituent band-gaps. At long wavelengths, the absorption spectra tend to the value of $\alpha_{\mathrm{i}}$, and the measured values are the same at both temperatures indicating that this parameter is not temperature-dependent within the experimental uncertainty of the measurement. Gain spectra measured at the two temperatures are also plotted in Figure 1. As is usually the case with semiconductor lasers, to obtain the same peak modal gain at the higher temperature requires a higher current density. However, rather than current density, which has contributions from a number of involved processes such as non-radiative recombination, we would prefer to use a parameter more closely related to the degree of population inversion. The well-known Bernard and Duraffourg condition ${ }^{4}$ states that population inversion (and therefore gain) cannot be achieved until the quasi-Fermi level separation $\left(\Delta \mathrm{E}_{\mathrm{f}}\right)$ exceeds the transition energy. At still higher pumping, the gain increases with the amount by which $\Delta \mathrm{E}_{\mathrm{f}}$ exceeds the transition energy. For a system in quasi-equilibrium the transparency point energy, where the modal gain is zero, determines $\Delta \mathrm{E}_{\mathrm{f}}$. We will make use of the difference between the transparency point and the transition energy as a measure of the degree of inversion of the dot material. For the inhomogeneously broadened dot states, it is difficult to identify a single transition energy, so we arbitrarily choose a magnitude of absorption on the absorption edge in Figure 1 to act as a common baseline for both temperatures as we have already observed that this rigidly shifts with increasing temperature. Comparing the gain spectra measured at $240 \mathrm{~K}$ and $300 \mathrm{~K}$ for the fixed value of absorption edge (AE) - transparency point (TP), we see they also show a rigid shift in the wavelength of peak gain, reflecting the temperature induced energy shift of the dot states seen in the absorption spectra. At the higher temperature, there is also a substantial decrease in gain magnitude at this fixed level of population inversion. We have previously reported on this effect, which is due to the increased thermal distribution of carriers amongst the available inhomogeneously broadened 
dot states, in both InP dots ${ }^{5}$ and InAs dots. ${ }^{1}$ Since in this experiment, $\alpha_{i}$ remains unchanged, the gain magnitude ( $\mathrm{G}=\alpha_{\mathrm{i}}+\alpha_{\mathrm{m}}$, where $\alpha_{\mathrm{m}}$ is the mirror loss) required for lasing is insensitive to temperature, because the mirror loss depends upon the reflectivity of the cleaved facets and the cavity length, both of which are relatively temperature-insensitive. Therefore, in order to maintain the necessary gain required to overcome the loss imposed by the cavity, the laser must be pumped to a greater degree of population inversion as the temperature increases to compensate for the decrease in gain magnitude at fixed IAE-TPI.

The increase in the number of carriers necessary to return the gain peak magnitude to the value to overcome the losses causes a blue-shift of the peak gain wavelength. This blue-shift can be large enough to cancel the red-shift caused by the temperature dependence of the band-gap and so the overall gain peak wavelength remains unchanged.

This effect is illustrated in Figure 1, where he dashed line indicates a gain spectrum measured at $300 \mathrm{~K}$, where IAE-TPI has been increased (by increasing the drive current) to a level necessary to recover the gain magnitude of the $240 \mathrm{~K}$ gain spectrum. The accompanying blue-shift moves the peak gain wavelength to a similar value as that of the $240 \mathrm{~K}$ spectrum, even though the shift of the underlying states is that of the bandgap.

To investigate the degree of state-filling further, we plot the gain spectra measured using the segmented contact method as a function of applied current density in Figure 2. A number of features are immediately apparent such as the broad gain spectrum and the fact that the gain peak wavelength blue-shifts, due to state-filling of the inhomogeneously broadened dot states, as the gain magnitude increases with increasing injection. In addition to the fundamental material properties, it is clear that the gain requirement, or equivalently the cavity loss, will determine the gain peak wavelength and, thus, the lasing wavelength in a simple edge-emitting laser diode. Such statements could also be made about quantum well lasers but the degree of blue-shift is substantially larger in this QD material.

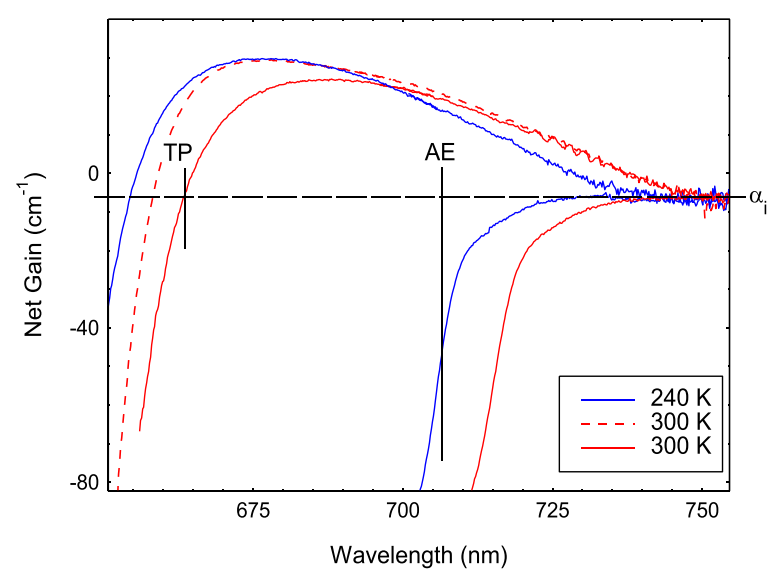

FIG. 1. The net modal gain and absorption spectra for a fixed degree of inversion (red and blue solid lines) at 240 and $300 \mathrm{~K}$. The shift in the $\mathrm{AE}$ reflects the change in the band gap energy with temperature and corresponds to a change of $0.17 \mathrm{~nm} / \mathrm{K}$. The dashed line shows the $300 \mathrm{~K}$ gain spectrum, when the inversion level |AE-TP| is increased to recover the fixed gain peak magnitude. The red-shift due to the temperature coefficient of the band-gap is compensated by the blue-shift of the peak due to state filling.

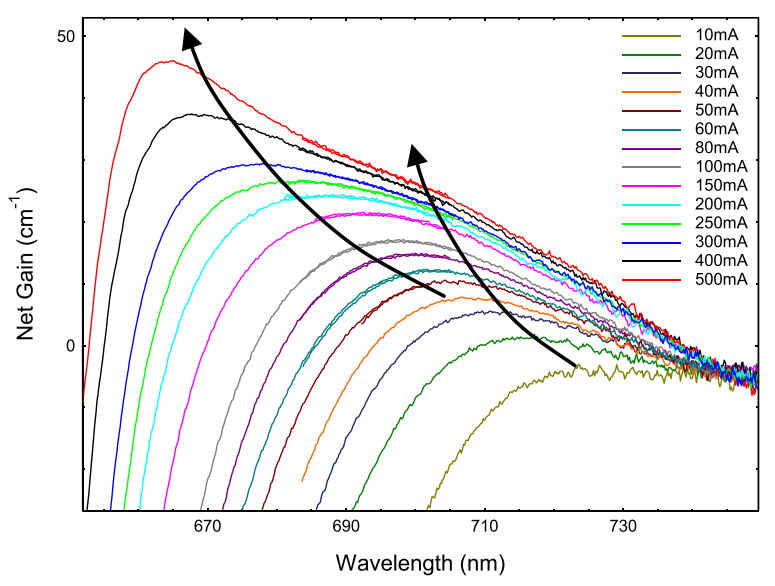

FIG. 2. Measured net modal gain spectra, for increasing injection current, at $300 \mathrm{~K}$. The arrows show how the gain spectrum evolves with progressive state-filling of the QD ensemble.

The blue-shift of the gain peak wavelength due to statefilling and therefore the temperature dependence of the overall gain peak wavelength depends on the absolute gain magnitude, and this is illustrated in Figure 3, where the measured change in wavelength with temperature $(\Delta \lambda / \Delta \mathrm{T})$ is plotted as a function of the peak net-gain $\left(\mathrm{G}-\alpha_{\mathrm{i}}\right)$. For a fixed gain at low injection, the wavelength dependence of the gain peak follows that of the band gap $(\approx 0.17 \mathrm{~nm} / \mathrm{K})$, as described by the Varshni equation, but at higher levels of injection the wavelength becomes relatively independent of operating temperature, until the gain increases beyond $30 \mathrm{~cm}^{-1}$. As described above, the size of $\Delta \lambda / \Delta \mathrm{T}$ is controlled by the thermal spreading of carriers in the dot states and the state-filling process, or more explicitly the relative extent to which the gain peak wavelength and the gain peak magnitude change as the level of pumping is increased. Starting from low population of the QD states at low levels of pumping, the gain peak magnitude can increase relatively rapidly with increasing pumping. The experimentally determined gain-current relations for a range of temperatures $(240-340 \mathrm{~K})$ are plotted in the inset of Figure 3. There are two distinct regions which correspond to

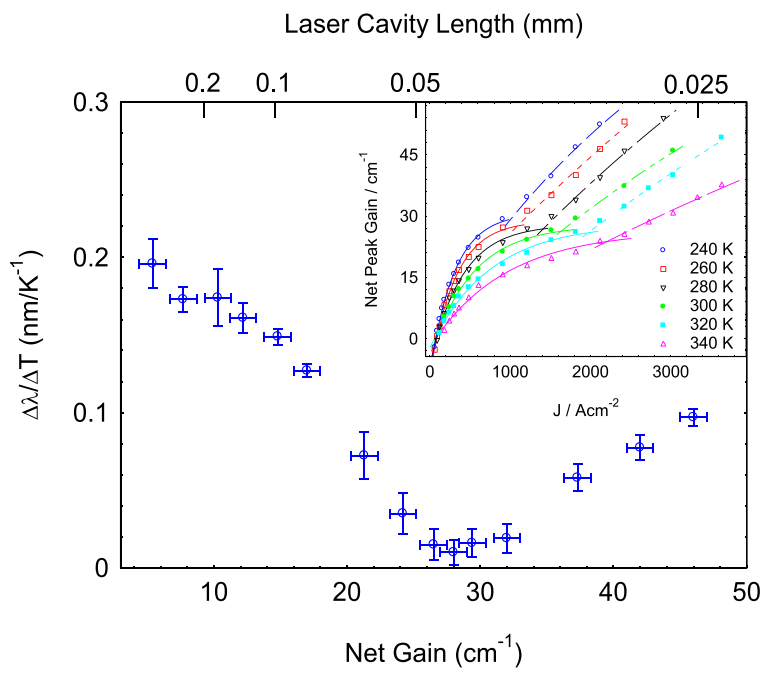

FIG. 3. The variation in the peak gain wavelength with temperature as a function of gain peak magnitude. Inset contains the gain-current relations for the sample measured at various temperatures. The two distinct regions are fitted separately using Eq. (1). 


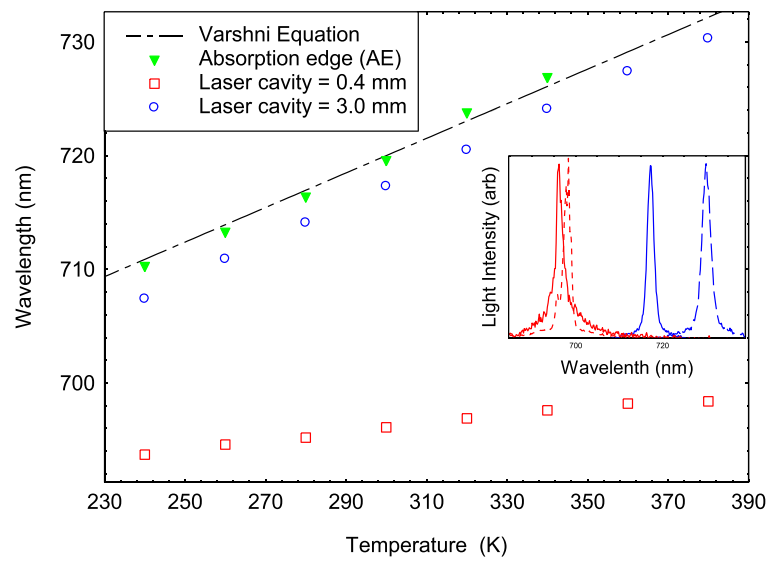

FIG. 4. Emission wavelength of edge-emitting lasers from 280 to $380 \mathrm{~K}$, together with the temperature dependence of the AE and the band-gap (calculated from the Varshni equation). Shown here is a laser with a $3 \mathrm{~mm}$ long cavity (circles) with $\Delta \lambda / \Delta \mathrm{T} \approx 0.17 \mathrm{~nm} / \mathrm{K}$ and a $0.4 \mathrm{~mm}$ long cavity with $\Delta \lambda / \Delta \mathrm{T} \approx 0.03 \mathrm{~nm} / \mathrm{K}$. Inset shows the spectra at $300 \mathrm{~K}$ (solid lines) and $380 \mathrm{~K}$ (dashed lines), for $3 \mathrm{~mm}$ (right) and $0.4 \mathrm{~mm}$ (left) length lasers.

carrier densities, where the maximum gain is achieved by either the ground or excited states. The data have been fitted empirically using ${ }^{6}$

$$
G=G_{0}\left[1-\exp \left(-\gamma \frac{J-J_{0}}{J_{o}}\right)\right],
$$

where $G_{0}$ is the gain parameter (asymptotic value of gain at infinite current), $J_{O}$ is the transparency current, and $\gamma$ is referred to as a ideality factor. As the inhomogeneously broadened dot ground states become highly populated the ground state gain magnitude tends to saturate, while the gain peak wavelength continues to shorten. At this point, the magnitude of $\Delta \lambda / \Delta \mathrm{T}$ is a minimum since the effect of state filling is maximised and this corresponds to region, where the fitted gain-current relations crossover. At still higher pumping, the largest gain is no longer obtained from the ground state and the larger number of excited states allows a more rapid increase in gain magnitude. In addition, the population of a high density of states decreases the change in quasi-Fermi level with increasing carrier injection and results in a relatively smaller change in the peak wavelength. This reduces the compensation of the bandgap-controlled wavelength shift and therefore results in larger value of $\Delta \lambda / \Delta \mathrm{T}$.

The data of Figure 3 suggest that for this material the laser designer should be able to select the temperature dependence of the lasing wavelength simply by selecting the cavity loss. In Figure 4, the peak wavelengths of edgeemitting lasers with cavity lengths of 0.4 and $3.0 \mathrm{~mm}$ are plotted for temperatures from $240-380 \mathrm{~K}$. Also plotted here are the temperature dependence of the bandgap, calculated from the Varshni equation, and the shift in the absorption edge with temperature. The $3.0 \mathrm{~mm}$ laser, with a mirror loss of $4 \mathrm{~cm}^{-1}$, exhibits a wavelength temperature dependence of $0.17 \mathrm{~nm} / \mathrm{K}$, which is very similar to the rate of change of the absorption edge and that calculated by the Varshni equation using input parameter appropriate for the GaInP barrier. However, as predicted by the gain measurements and the summary in Figure 3, the $0.4 \mathrm{~mm}$ laser, with an $\alpha_{\mathrm{m}}$ of $30 \mathrm{~cm}^{-1}$, exhibits a change in lasing wavelength of only $0.03 \mathrm{~nm} / \mathrm{K}$, which is almost identical to the wavelength shift of a Bragg stack fabricated in this material.

Since the mirror loss increases as the length of the cavity becomes smaller, the reduced wavelength temperature sensitivity comes at the expense of an increase in the threshold current density $\left(J^{\text {th }}\right)$. The super-linear increase in $J^{\text {th }}$ that occurs in the range of $240-380 \mathrm{~K}$ is $3.3 \mathrm{KAcm}^{-2}$ and 475 $\mathrm{Acm}^{-2}$ for a 0.4 and $3.0 \mathrm{~mm}$ long cavity, respectively. At $380 \mathrm{~K}$ the driving current for both devices are similar, at 800 and $850 \mathrm{~mA}$.

While the results reported here are specific to a type of InP QD laser material, we expect similar results to be obtained for all types of QD materials, albeit at a different level of pumping, because the origin of the effect is manifested in the way carriers populate the inhomogeneously broadened QD states.

We have identified the mechanisms which govern the temperature dependence of the gain peak wavelength for an InP/AlGaInP QD ensemble and have identified the regime in which it can be temperature-insensitive. This work demonstrates that the effect is a general feature of inhomogeneously broadened QD materials. We have shown that it can be easily exploited to produce edge-emitting lasers with a relatively temperature-insensitive operating wavelength.

${ }^{1}$ S. N. Elliott, P. M. Smowton, A. B. Krysa, and R. Beanland, Semicond. Sci. Technol. 27, 094008 (2012).

${ }^{2}$ P. M. Smowton, I. C. Sandall, H. Y. Liu, and M. Hopkinson, J. Appl. Phys. 101, 013107 (2007).

${ }^{3}$ P. Blood, G. M. Lewis, P. M. Smowton, H. Summers, J. Thomson, and J. Lutti, IEEE J. Sel. Top. Quantum Electron. 9(5), 1275-1282 (2003).

${ }^{4}$ M. G. A. Bernard and G. Duraffourg, Phys. Status Solidi B 1, 699 (1961).

${ }^{5}$ P. M. Smowton, S. N. Elliott, S. Shutts, M. S. Al-Ghamdi, and A. B. Krysa, IEEE J. Sel. Top. Quantum Electron. 17(5), 1343-1348 (2011).

${ }^{6}$ V. M. Ustinov, A. E. Zhukov, A. Y. Egorov, and N. A. Maleev, Quantum Dot Lasers (Oxford University Press, Oxford, 2003). 